\title{
A CATEGORICAL APPROACH TO MATRIX TODA BRACKETS
}

\author{
K. A. HARDIE, K. H. KAMPS AND H. J. MARCUM
}

\begin{abstract}
In this paper we give a categorical treatment of matrix Toda brackets, both in the pre- and post-compositional versions. Explicitly the setting in which we work is, à la Gabriel-Zisman, a 2-category with zeros. The development parallels that in the topological setting but with homotopy groups replaced by nullity groups of invertible 2-morphisms. A central notion is that of conjugation of 2-morphisms. Our treatment of matrix Toda brackets is carried forward to the point of establishing appropriate indeterminacies.
\end{abstract}

\section{INTRODUCTION}

When P. Gabriel and M. Zisman wrote their book [3] they presented their subject (homotopy theory of simplicial sets via categories of fractions) from the most general point of view available going "back to the beginning of the theory ... in the hope of providing the reader with ... proofs which are easier or more conceptual than those already published." Their study of homotopy having begun in Chapter IV they set out in Chapter V "to give a standard and self-dual proof of various exact sequences occurring in Algebraic Topology ... the main idea is to construct an exact sequence in the category of pointed groupoids and then to reduce the study of a large class of 2-categories to the preceding one." They soon specialise to 2-categories $\mathscr{Z}$ which satisfy conditions $\mathrm{A}$ and $\mathrm{B}$ below :

A. If $x$ and $y$ are two objects of $\mathscr{Z}, \operatorname{Hom}_{\mathscr{Z}}(x, y)$ is a groupoid.

B. There is an object 0 of $\mathscr{Z}$ such that, for any object $x$ of $\mathscr{Z}$, $\operatorname{Hom}_{\mathscr{L}}(o, x)$ and $\operatorname{Hom}_{\mathscr{L}}(x, o)$ are isomorphic to the zero category O.

Continuing in the spirit of Gabriel and Zisman the present paper is a contribution to an abstract theory of homotopy that assumes the existence of a 2-category $\mathscr{C}$ satisfying only condition $\mathrm{B}$. Indeed it can be regarded as sequel to a paper [8] by the third author which, in the setting of an arbitrary 2-category, studies the notion of homotopy equivalence and characterises the homotopy equivalences in various lax categories. Here the development parallels that in the topological setting but with track groups of the form $\pi(\Sigma X, Y)$ or $\pi(X, \Omega Y)$ replaced by nullity groups of invertible 2-morphisms. A central notion is that of conjugation of 2-morphisms. This notion is available in any 2-category but does not seem to have been used previously. In fact when

Received by the editors June 9, 1993.

1991 Mathematics Subject Classification. Primary 18D05, 55Q05, 55Q35.

(C)1995 American Mathematical Society 
dealing with a groupoid enriched category we show that the homotopy category and the category of conjugacy classes are isomorphic categories. But the main objective is to show that the new homotopy theory also can be equipped with secondary composition operations modulo certain indeterminacy, and to compute the indeterminacy in the case of the abstract analogues of the Toda brackets, including matrix Toda brackets. Since we consider post-compositional as well as pre-compositional matrix Toda brackets the discussion of the indeterminacy (in abstract form) may be more complete than in the existing literature where results are often stated (for simplicity) in special cases (cf. [4]). Some of the formulae (see Theorems 5.5, 7.1) look slightly different to corresponding results in the topological case but, when appropriately interpreted, they do specialise correctly. Other formulae are new (see Theorem 5.4).

Computing the operations in particular 2-categories will certainly be difficult. Perhaps the most promising approach would be to exploit the methods of BauesDreckmann [2]. However we do not consider here the problem of computation. The results show that a theory of matrix Toda brackets is also available in the injective (respectively projective) homotopy theory of modules over a ring described by Eckmann and Hilton [6]. The category of pairs and homotopy pair maps [5] can be equipped with the structure of a 2-category with zeros and it is reasonable to believe that a study of the Toda bracket in this category (and other similar diagram categories) may throw light on the properties (and definition) of the classical higher order Toda brackets in the topological case.

As a further historical point, in the topological category matrix Toda brackets (in the post-compositional version only) were first mentioned by Barratt [1] and then by Mimura [10]. Both of these authors restricted themselves to the "stable setting" in which the initial space of the square defining the relation is assumed to be a suspension space. This restriction allows the operation to be regarded as a special case of the Toda bracket. Our paper [4] defined the pre-compositional version of matrix Toda brackets in the topological category for the more general unstable setting. In the present paper of course we always work, by necessity, in an unstable framework.

\section{CONJUGATION OF 2-MORPHISMS}

(1.1) Definition. Let $\mathscr{C}$ be a 2-category. For objects $A$ and $B$ of $\mathscr{C}$ suppose that $\sim_{A, B}$ denotes an equivalence relation on the 2-morphisms of the category $\operatorname{hom}_{\mathscr{E}}(A, B)$. For a given 2-morphism $F: f \Rightarrow g: A \rightarrow B$ let

$$
\langle F\rangle=\{\overbrace{\underbrace{A G}_{k} \underbrace{}_{B} \mid G \sim_{A, B} F}^{h}\}
$$

denote its equivalence class. Now if the given equivalence relations are compatible with the horizontal composition of 2-morphisms, in the sense that if $F \sim_{A, B} F^{\prime}$ and $J \sim_{B, C} J^{\prime}$ then $J \circ F \sim_{A, C} J^{\prime} \circ F^{\prime}$, then one can associate a category to these equivalence relations. This category has the same objects as $\mathscr{C}$ and the morphisms are the equivalence classes $\langle F\rangle: A \rightarrow B$. Further 
note that assigning to the 1-morphism $f: A \rightarrow B$ of $\mathscr{C}$ the equivalence class $\left\langle 1_{f}\right\rangle: A \rightarrow B$ defines a functor from $\mathscr{C}$ to this associated category.

\section{(1.2) Definition. Let}

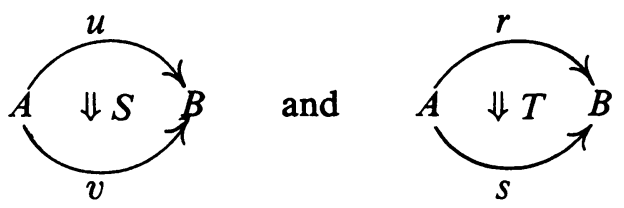

be 2-morphisms of a 2-category $\mathscr{C}$. We say that $S$ and $T$ are conjugate and write $S\langle$ conj $\rangle T$ if there exist invertible 2-morphisms $H: v \Rightarrow s$ and $K: u \Rightarrow r$ such that $H+S=T+K$.

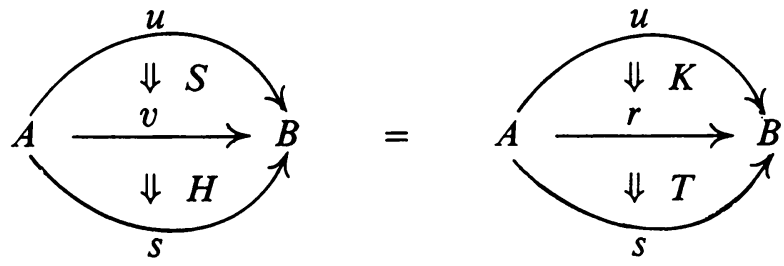

It is straightforward to verify that $\langle c o n j\rangle$ is an equivalence relation on the 2-morphisms of each category $\operatorname{hom}_{\mathscr{C}}(A, B)$ and that it is compatible with the horizontal composition of 2-morphisms. Hence by Definition 1.2 there is an associated category which we denote $\mathscr{C}^{<>}$and call the category of conjugacy classes of $\mathscr{C}$.

(1.3) Proposition. (a) If $S: u \Rightarrow v$ is invertible then $\langle S\rangle=\langle 1 u\rangle=\langle 1 v\rangle$.

(b) If $S\langle$ con $j\rangle T$ as in Definition 1.2 and $S$ is invertible then $T$ is invertible.

Proof. (a) When $S$ is invertible the equation $S+1 v=1 u+S$ implies both that $S\langle$ con $j\rangle 1 u$ and that $S\langle c o n j\rangle 1 v$.

(b) The equation $H+S=T+K$ can be rewritten $H+S-K=T$ with each of $H, S$ and $K$ invertible. Hence $T$ must be invertible.

(1.4) Definition. Let $\mathscr{B}$ be a 2-category. Two 1-morphisms $f, g: A \rightarrow B$ of $\mathscr{C}$ are said to be homotopic if there exists an invertible 2-morphism $F: f \Rightarrow g$. We write $f \simeq g$. The relation $\simeq$ is an equivalence relation on the 1 -morphisms of $\mathscr{C}$ and is compatible with the compositon of 1 -morphisms. Hence we may form the quotient category of $\mathscr{C}$ by the equivalence relation $\simeq ;$ this is denoted $\mathrm{H} \mathscr{C}$ and called the homotopy category. The morphisms of $\mathrm{H} \mathscr{C}$ are homotopy classes [f]: $A \rightarrow B$ and there is a projection functor proj: $\mathscr{C} \rightarrow \mathrm{H} \mathscr{C}$.

Note that in the above definition of homotopic we have avoided needing to assume that $\mathscr{C}$ be groupoid enriched (as done for example in [7] and [11]) by simply requiring $F$ to be invertible.

Observe by Proposition 1.3.a that if $f \simeq g: A \rightarrow B$ then $1 f\langle$ conj $\rangle 1 g$. It follows that there is an induced functor $\mathrm{H} \mathscr{C} \rightarrow \mathscr{C}^{<>}$given by $[f] \mapsto\left\langle 1_{f}\right\rangle$. The composite functor

$$
\mathscr{C} \stackrel{\text { proj }}{\longrightarrow} \mathrm{H} \mathscr{C} \longrightarrow \mathscr{C}^{<>}
$$

is just the induced functor mentioned in Definition 1.1 .

We recall that a groupoid enriched category is a 2-category in which each 2-morphism is invertible. 
(1.5) Theorem. If $\mathscr{C}$ is a groupoid enriched category then the functor

$$
\mathbf{H} \mathscr{C} \longrightarrow \mathscr{C}<>\quad, \quad[f] \mapsto\left\langle 1_{f}\right\rangle,
$$

is an isomorphism of categories.

Proof. A functor $\mathscr{C}<>\rightarrow \mathrm{H} \mathscr{C}$ may be specified by the assignment $\langle F\rangle \mapsto[f]$ where $F: f \Rightarrow g$. This assignment is well-defined because if $F: f \Rightarrow g$ is invertible then $\langle F\rangle=\langle 1 f\rangle=\langle 1 g\rangle$ and $[f]=[g]$. Since clearly each of the composites

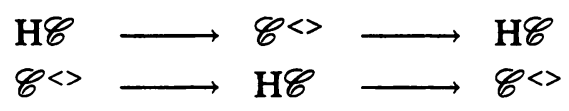

is the identity functor the result follows.

\section{THE NULLITY OPERATOR}

In this section we suppose that $\mathscr{C}$ is a 2-category which satisfies Condition B (but not necessarily Condition A) on page 81 of [3]. Such a $\mathscr{E}$ is said to be a 2-category with zeros. In particular each of the categories $\operatorname{hom}_{\mathscr{C}}(A, B)$ is provided with a distinguished zero 1-morphism denoted $o: A \rightarrow B$.

Definition. If

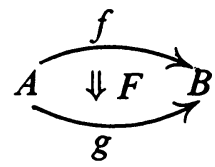

is a 2-morphism of $\mathscr{C}$ we define the nullity of $F$ by

$$
\mathrm{N}(F)=\{\overbrace{A_{0} \frac{O}{\Downarrow G}}^{B} \mid G\langle c o n j\rangle F\} .
$$

Observe that $\mathrm{N}(F) \subset\langle F\rangle$.

(2.2) Proposition. Let $F: f \Rightarrow g$ be a 2-morphism in $\mathscr{C}$. Then the following properties hold.

(a) $\mathrm{N}(F)$ is nonvacuous if and only if $f \simeq 0$ and $g \simeq 0$.

(b) If $F\langle$ con.j $\rangle F^{\prime}$ then $\mathrm{N}(F)=\mathrm{N}\left(F^{\prime}\right)$.

(c) If $F$ is invertible then $\mathrm{N}(F)=\mathrm{N}(1 f)=\mathrm{N}(1 g)$.

(d) If $f \simeq g: A \rightarrow B$ then $\mathrm{N}\left(1_{f}\right)=\mathrm{N}\left(1_{g}\right)$.

(e) If $G^{\prime}: o \Rightarrow o$ is invertible then $G+G^{\prime}$ and $G^{\prime}+G$ belong to $\mathrm{N}(F)$ if $G$ does.

Proof. Properties (a)-(d) follow easily from the definition of $\mathrm{N}(F)$ and we omit their proofs. To verify part (e), let $G: o \Rightarrow o$ be in $\mathrm{N}(F)$ and choose invertible 2-morphisms $H: o \Rightarrow f$ and $K: o \Rightarrow g$ satisfying $K+G=F+H$. Since we assume that $G^{\prime}$ is invertible so also is the composite 2-morphism $H+G^{\prime}$. Then the equation $K+\left(G+G^{\prime}\right)=F+\left(H+G^{\prime}\right)$ shows that $\left(G+G^{\prime}\right)\langle$ conj $\rangle F$, as claimed. In a similar way one shows that $G^{\prime}+G \in$ $\mathrm{N}(F)$. 
(2.3) Definition. If $f: A \rightarrow B$ is a 1 -morphism in $\mathscr{C}$, we denote by $\mathscr{A}(f)$ the set of all invertible 2-morphisms $f \Rightarrow f$. Plainly $\mathscr{A}(f)$ is a group under vertical composition of 2-morphisms with group identity $1, f: f \Rightarrow f$.

(2.4) Proposition. $A$ 2-morphism $\xi: f \Rightarrow f: A \rightarrow B$ is invertible if and only if $\xi$ is conjugate to $1_{f}$. That is, $\mathscr{A}(f)=\left\{\xi: f \Rightarrow f \mid \xi\langle\right.$ con $\left.j\rangle 1_{f}\right\}$. Note in particular that $\mathscr{A}(o: A \rightarrow B)=\mathrm{N}\left(1_{o}: o \Rightarrow o: A \rightarrow B\right)$.

Proof. If $\xi$ is invertible then the identity equation $1_{f}+\xi=1_{f}+\xi$ shows that $\xi\langle c o n j\rangle 1_{f}$. On the other hand, if $\xi$ is conjugate to $1_{f}$ then by Proposition 1.3.b $\xi$ must be invertible, because $1_{f}$ is invertible.

(2.5) Proposition. Suppose that $F: f \Rightarrow g: A \rightarrow B$ is an invertible 2-morphism of $\mathscr{C}$. If $\mathrm{N}(F)$ is nonvacuous then it coincides with $\mathscr{A}(o: A \rightarrow B)$. In particular, in this case, $\mathrm{N}(F)$ is a group under the vertical compositon of 2-morphisms in the category hom $\mathscr{C}(A, B)$; the group identity of $\mathrm{N}(F)$ is the identity 2-morphism $1_{o}: o \Rightarrow o$. Note moreover that, in this case, $\mathrm{N}(F)$ does not depend upon $F$.

Proof. Firstly, since $F$ is invertible, $\mathrm{N}(F)=\mathrm{N}\left(1_{f}\right)$ by Proposition 2.2.c. And since $\mathrm{N}(F)$ is nonvacuous, Proposition 2.2.a implies that $f \simeq 0$ and consequently $\mathrm{N}\left(1_{f}\right)=\mathrm{N}\left(1_{O}\right)$ by Proposition 2.2.d. It follows that $\mathrm{N}(F)=\mathrm{N}\left(1_{O}\right)$. But, by Proposition 2.4, $\mathrm{N}\left(1_{O}\right)=\mathscr{A}(o: A \rightarrow B)$ and the latter is a group. This establishes the proposition.

Let $F$ and $H$ be 2-morphisms in $\mathscr{C}$ as indicated:

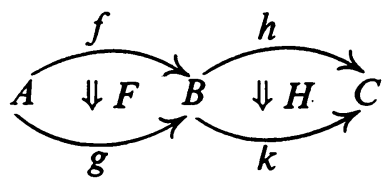

We denote by $H \circ F$ (or sometimes just $H F$ ) the horizontal composition of $F$ and $H$. We also write $h \circ F$ or $h F$ instead of $1_{h} \circ F$ and $H \circ f$ or $H f$ instead of $H \circ 1_{f}$. Note that if $F$ and $H$ are invertible then so in $H \circ F$. Furthermore by the interchange law for horizontal and vertical composition the equation

$$
k F+H f=H g+h F
$$

is valid. Consequently we obtain

(2.6) Proposition. Consider the 2-morphisms $F$ and $H$ given above. If $F$ is invertible then $H f\langle c o n j\rangle H g$, and if $H$ is invertible then $h F\langle c o n j\rangle k F$.

(2.7) Definition. Suppose given the data:

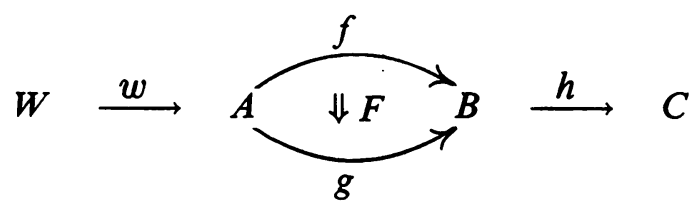

Then there are induced functions

$$
\begin{aligned}
& \mathrm{N}(F) \longrightarrow \mathrm{N}(h F), \\
& \mathrm{N}(F) \longrightarrow \mathrm{N}(F w), \\
& \quad G \mapsto G \mapsto G,
\end{aligned}
$$


where $G \in \mathrm{N}(F)$. Note that these functions are well-defined since conjugation of 2-morphisms is compatible with horizontal composition. If $\mathscr{E} \subset \mathrm{N}(F)$ is a subset, its images under these functions will be denoted by $h \circ \mathscr{E}$ and $\mathscr{E} \circ w$ respectively.

(2.8) Proposition. (a) If $h: B \rightarrow C$ is a homotopy equivalence in $\mathscr{C}$ then the induced function $\mathrm{N}(F) \rightarrow \mathrm{N}(h F)$ is a bijection.

(b) If $w: W \rightarrow A$ is a homotopy equivalence in. $\mathscr{C}$ then the induced function $\mathrm{N}(F) \rightarrow \mathrm{N}(F w)$ is a bijection.

Proof. We only prove part (a); the proof for part (b) is similar. Let $\bar{h}: C \rightarrow B$ be a homotopy inverse for $h$. Thus there are invertible 2-morphisms $U: \bar{h} h \Rightarrow 1_{B}$ and $V: h \bar{h} \Rightarrow{ }^{1} C$. By Vogt's Lemma [8, Lemma 1.5] we may assume that $h U=V h$ and $U \bar{h}=\bar{h} V$.

Let $L: o \Rightarrow o$ in $\mathrm{N}(h F)$ be given. Denote by $K$ the composite 2-morphism:

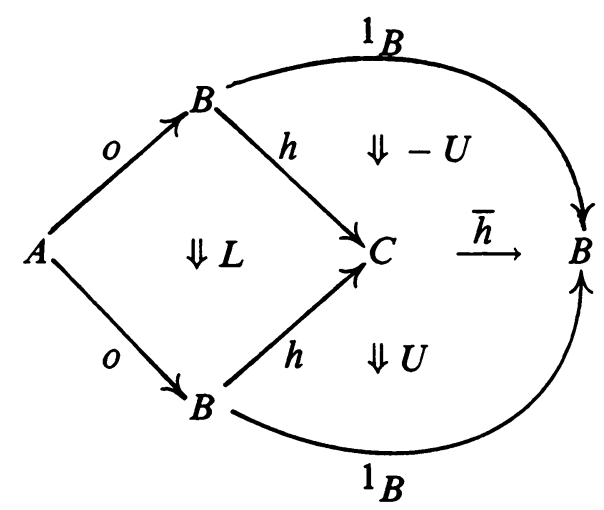

Because $h U=V h$ it follows at once that $h K=L$. Moreover $K$ belongs to $\mathrm{N}(F)$ as can be seen by observing that the chain of relations

$$
K\langle c o n j\rangle \bar{h} L\langle c o n j\rangle \bar{h} h F\langle\text { con } j\rangle F
$$

is valid. The first of these relations, $K\langle c o n j\rangle \bar{h} L$, follows directly by the definition of $K$. Also by assumption $L\langle$ conj $\rangle h F$ and hence $\bar{h} L\langle$ conj $\rangle \bar{h} h F$. And the last relation $\bar{h} h F\langle$ con $j\rangle F$ follows by Proposition 2.6 since $\bar{h} h \simeq 1_{B}$. Thus surjectivity of $\mathrm{N}(F) \rightarrow \mathrm{N}(h F)$ is established.

Finally we verify that $\mathrm{N}(F) \rightarrow \mathrm{N}(h F)$ is injective. First observe that

$$
U+{ }^{1} \bar{h} h-U=1_{1} \text {. }
$$

Hence, by use of the interchange law, for any $G \in \mathrm{N}(F)$ we obtain

$$
G=1_{B} \circ G=\left(U+1_{\bar{h} h}-U\right) \circ G=U \circ 1_{o}+\bar{h}(h G)-U \circ 1_{o} .
$$

But from this last equation it is immediate that if $G_{1}, G_{2} \in \mathrm{N}(F)$ satisfy $h G_{1}=h G_{2}$ then $G_{1}=G_{2}$. Thus $\mathrm{N}(F) \rightarrow \mathrm{N}(h F)$ is injective and consequently a bijection.

(2.9) Proposition. Let the 2-morphism $F$ given in Definition 2.7 be invertible. If $\mathrm{N}(F)$ is nonvacuous then the functions $\mathrm{N}(F) \rightarrow \mathrm{N}(h F)$ and $\mathrm{N}(F) \rightarrow \mathrm{N}(F w)$ are homomorphisms of groups. 
Proof. If $G_{1}$ and $G_{2}$ are in $\mathrm{N}(F)$ then by use of the interchange law

$$
\begin{aligned}
h\left(G_{1}+G_{2}\right) & =1_{h} \circ\left(G_{1}+G_{2}\right) \\
& =\left(1_{h}+1_{h}\right) \circ\left(G_{1}+G_{2}\right) \\
& =\left(1_{h} \circ G_{1}\right)+\left(1_{h} \circ G_{2}\right) \\
& =h G_{1}+h G_{2}
\end{aligned}
$$

so that $\mathrm{N}(F) \rightarrow \mathrm{N}(h F)$ is indeed a group homomorphism. Similarly we see that $\mathrm{N}(F) \rightarrow \mathrm{N}(F w)$ is a group homomorphism.

(2.10) Remark. It should be pointed out that the functions in Definition 2.7 do depend on the 1-morphisms $h$ and $w$ and not just on their homotopy classes. For example, if $h \simeq h^{\prime}$ then indeed $\mathrm{N}(h F)=\mathrm{N}\left(h^{\prime} F\right)$ by Proposition 2.6 and Proposition 2.2.b but it need not be true even that $h \circ \mathrm{N}(F)=h^{\prime} \circ \mathrm{N}(F)$.

\section{THE $\sigma$-OPERATIONS}

(3.1) Definition. Let $\mathscr{C}$ be a 2-category with zeros. Suppose given the following data in $\mathscr{C}$ :

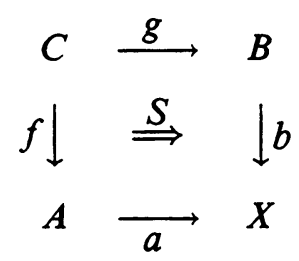

Then for a 1-morphisms $w: W \rightarrow C$ we define a subset $\sigma(S, w) \subset \mathrm{N}(S w)$ by

$$
\sigma(S, w)=\{-b K+S w+a H \mid H: o \Rightarrow f w,
$$

$K: o \Rightarrow g w$ and $H$ and $K$ are invertible $\}$

and for a 1-morphism $x: X \rightarrow Y$ we define a subset $\sigma(x, S) \subset \mathrm{N}(x S)$ by

$$
\sigma(x, S)=\left\{-V g+x S+U f \mid \begin{array}{l}
\mid \\
V: o \Rightarrow x a, \\
V: o \Rightarrow x b \text { and } U \text { and } V \text { are invertible }\} .
\end{array}\right.
$$

Clearly $\sigma(S, w)$ is nonvacuous if and only if $f w \simeq 0$ and $g w \simeq 0$. Similarly $\sigma(x, S)$ is nonvacuous if and only if $x a \simeq 0$ and $x b \simeq 0$. When $\sigma(S, w)$ and $\sigma(x, S)$ are nonvacuous we say that they are defined.

It should be pointed out that the notations $\sigma(S, w)$ and $\sigma(x, S)$ do not reflect the structure of the 2-morphism $S$ as a "square" in terms of the 1morphisms $f, g, a$ and $b$. In this sense these notations are insufficient. We will clarify this aspect of these operations in the next section.

(3.2) Proposition. In Definition 3.1 suppose that $S$ is an invertible 2-morphism. Then $\sigma(-S, w)=-\sigma(S, w)$ and $\sigma(x,-S)=-\sigma(x, S)$.

Proof. If $S: a f \Rightarrow g b$ is invertible then $-(-b k+S w+a H)=-a H-S w+b K$ from which the equality $-\sigma(S, w)=\sigma(-S, w)$ follows. Similarly for the second equality.

(3.3) Proposition. (i) If $w \simeq \bar{w}: W \rightarrow C$ then $\sigma(S, w)=\sigma(S, \bar{w})$. 
(ii) If $x \simeq \bar{x}: X \rightarrow Y$ then $\sigma(x, S)=\sigma(\bar{x}, S)$.

Proof. We only prove part (i); the proof of part (ii) is similar. Because $w \simeq \bar{w}$ it is immediate that $\sigma(S, w)$ is defined if and only if $\sigma(S, \bar{w})$ is defined. Now fix an invertible 2-morphism $F: w \Rightarrow \bar{w}$ and suppose that $-b K+S w+a H$ is a 2-morphism in $\sigma(S, w)$. Here $H: O \Rightarrow f w$ and $K: O \Rightarrow g w$ are assumed to be invertible 2-morphisms. Hence the composite 2-morphisms $f F+H: 0 \Rightarrow f \bar{w}$ and $g F+K: 0 \Rightarrow g \bar{w}$ are also invertible. Then by use of the interchange law for vertical and horizontal composition in $\mathscr{C}$ we compute

$$
\begin{aligned}
-b K+S w+a H & =-b K+S \circ 1 w+a H \\
& =-b K+S \circ(-F+1 \bar{w}+F)+a H \\
& =-b K-b g F+S \bar{w}+a f F+a H \\
& =-b(g F+K)+S w+a(f F+H) .
\end{aligned}
$$

Since

$$
-b(g F+K)+S w+a(f F+H)
$$

is a 2-morphism in $\sigma(S, \bar{w})$ we conclude that $\sigma(S, w) \subset \sigma(S, \bar{w})$. Mutatis mutandis $\sigma(S, w) \subset \sigma(S, \bar{w})$. Thus $\sigma(S, w)=\sigma(S, \bar{w})$.

(3.4) Theorem. In Definition 3.1 suppose that $S$ is invertible. Then

(i) in the group $\mathrm{N}(S w)$ the subset $\sigma(S, w)$ is, if defined, a double coset of the subgroups $b \circ \mathrm{N}(1 g w)$ and $a \circ \mathrm{N}(1 f w)$, and

(ii) in the group $\mathrm{N}(x S)$ the subset $\sigma(x, S)$ is, if defined, a double coset of the subgroups $\mathrm{N}\left(1_{x b}\right) \circ g$ and $\mathrm{N}\left(1_{x a}\right) \circ f$.

Proof. We only prove part (i); the proof of part (ii) is similar. Now since $S$ is invertible so is $S w$; hence by Proposition 2.2.c we have

$$
\mathrm{N}(S w)=\mathrm{N}\left(1_{a f w}\right)=\mathrm{N}\left(1_{b g w}\right) \text {. }
$$

Consequently $a \circ \mathrm{N}\left(1_{f w}\right) \subset \mathrm{N}\left(1_{a f w}\right)$ and $b \circ \mathrm{N}\left(1_{g w}\right) \subset \mathrm{N}\left(1_{b g w}\right)$ are indeed subgroups of $\mathrm{N}(S w)$. Let $\theta=-b K+S w+a H$ be a fixed but arbitrary element of $\sigma(S, w)$. Here of course $H: 0 \Rightarrow f w$ and $K: 0 \Rightarrow g w$ are invertible 2morphisms of $\mathscr{C}$. We shall prove (i) by establishing the equality:

$$
\sigma(S, w)=b \circ \mathrm{N}(1 g w)+\theta+a \circ \mathrm{N}\left(1_{f w}\right)
$$

Let $G: 0 \Rightarrow o$ belong to $\mathrm{N}(1 g w)$ and $L: O \Rightarrow o$ belong to $\mathrm{N}\left(1_{f w}\right)$. By Proposition 1.3.b each of $G$ and $L$ must be invertible. Then

$$
b G+\theta+a L=b G-b K+S w+a H+a L=-b(K-G)+S w+a(H+L)
$$

is an element of $\sigma(S, w)$ since $K-G: o \Rightarrow g w$ and $H+L: o \Rightarrow f w$ are invertible 2-morphisms of $\mathscr{C}$. This shows that the inclusion

$$
b \circ \mathrm{N}(1 g w)+\theta+a \circ \mathrm{N}\left(1_{f} w\right) \subset \sigma(S, w)
$$

is valid.

Finally, to verify the reverse inclusion, let $-b K^{\prime}+S w+a H^{\prime}$ be any element of $\sigma(S, w)$ with $H^{\prime}: 0 \Rightarrow f w$ and $K^{\prime}: 0 \Rightarrow g w$ invertible 2-morphisms of $\mathscr{C}$. Then we may write

$$
\begin{aligned}
-b K^{\prime}+S w+a H^{\prime} & =b\left(-K^{\prime}+K\right)-b K+S w+a H+a\left(-H+H^{\prime}\right) \\
& =b\left(-K^{\prime}+K\right)+\theta+a\left(-H+H^{\prime}\right)
\end{aligned}
$$


Since $-H+H^{\prime}: o \Rightarrow o$ belongs to $\mathrm{N}\left(1_{f w}\right)$ and $-K^{\prime}+K: 0 \Rightarrow o$ belongs to $\mathrm{N}(1 g w)$ it follows that we have represented $-b K^{\prime}+S w+a H^{\prime}$ as an element of $b \circ \mathrm{N}(1 g w)+\theta+a \circ \mathrm{N}\left(1_{f w}\right)$, as desired.

We refer to the double cosets given in Theorem 3.4 as the indeterminacies of the $\sigma$-operations. Further we say that the indeterminacy of a given operation is trivial if the subgroups involved are trivial. As an example consider the following diagram

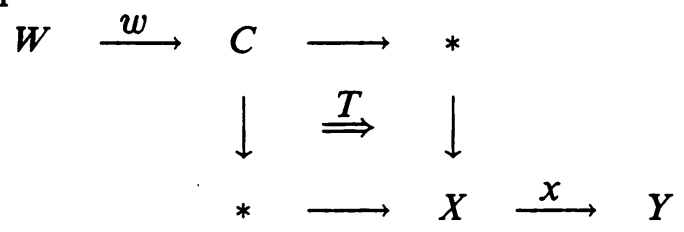

in which $*$ denotes the zero object of $\mathscr{C}$. Since $\operatorname{hom}_{\mathscr{C}}(W, *)$ and hom $_{\mathscr{C}}(*, Y)$ are the zero categories the next proposition is immediate.

(3.6) Proposition. Let notation be as in diagram (3.5). Then

(a) $\sigma(T, w)$ is defined with trivial indeterminacy and consists of the single element $T w: o \Rightarrow 0$, and

(b) $\sigma(x, T)$ is defined with trivial indeterminacy and consists of the single element $x T: 0 \Rightarrow 0$.

\section{NATURALITY}

In the definitions of $\sigma(S, w)$ and $\sigma(x, S)$ it is required that $S$ be a "square" in $\mathscr{C}$. We make this aspect of these definitions explicit by utilizing the category $\mathscr{Q}(\mathscr{C})$ of lax squares of $\mathscr{C}$, as described for example in $[9, \S 2]$ We recall that $\mathscr{Q}(\mathscr{C})$ is itself a 2-category. The objects of $\mathscr{Q}(\mathscr{C})$ are squares

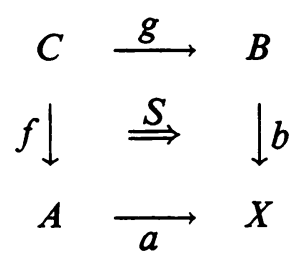

in which $S: a f \Rightarrow b g$ is a 2 -morphism of $\mathscr{C}$. The 1 -morphisms of $\mathscr{Q}(\mathscr{C})$ are cubes

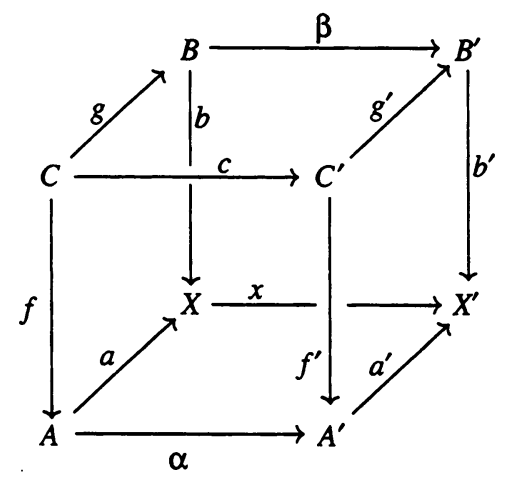


in which each square that is a face of the cube is provided with a 2-morphism (these are unlabelled in the cube but specified below ) so as to satisfy the coherence condition that the two composite 2 -morphisms of $\mathscr{C}$ represented by the following diagrams are equal.
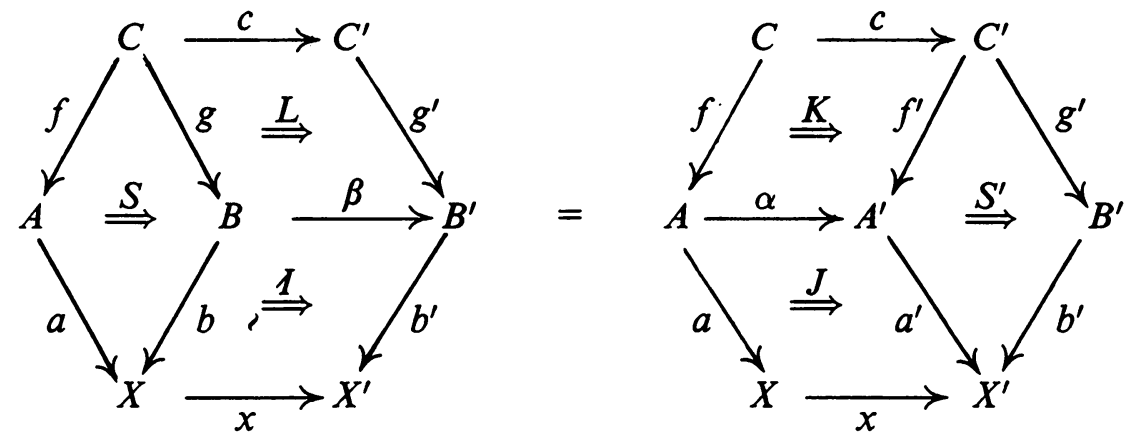

$$
b^{\prime} L+I g+x S=S^{\prime} c+a^{\prime} K+J f
$$

The left face (containing $S$ ) of the cube (4.1) is its domain as a 1-morphism of $\mathscr{Q}(\mathscr{C})$ and its right face (containing $S^{\prime}$ ) is its codomain. The 2-morphisms of $\mathscr{Q}(\mathscr{C})$ are modifications of the cubes $(4.1)$. To describe such a modification let the two cubes (or 1-morphisms) of $\mathscr{Q}(\mathscr{C})$ indicated below have the same left faces and the same right faces.
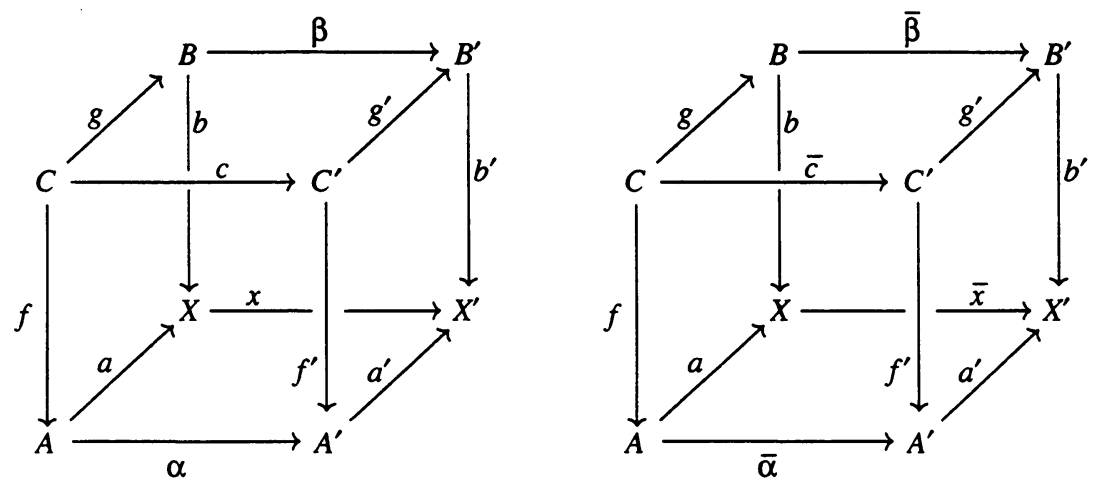

Then a modification (or 2-morphism) in $\mathscr{Q}(\mathscr{C})$ between these two cubes consists of four 2-morphisms of $\mathscr{C}$

$$
R: c \Rightarrow \bar{c}, \quad V: \beta \Rightarrow \bar{\beta}, \quad T: \alpha \Rightarrow \bar{\alpha}, \quad U: x \Rightarrow \bar{x}
$$

satisfying the equations $g^{\prime} R+L=\bar{L}+V g$ and $a^{\prime} T+J=\bar{J}+U a$.

The next proposition follows immediately from the coherence equation (4.2).

(4.3) Proposition. Suppose that the 1-morphism of $\mathscr{Q}(\mathscr{C})$ given by (4.1) has the additional property that $L, I, K$ and $J$ are invertible 2-morphisms of $\mathscr{C}$. Then $x S\langle$ con $j\rangle S^{\prime} c$ and consequently $\mathrm{N}(x S)=\mathrm{N}\left(S^{\prime} c\right)$.

By [8, Theorem 2.5] we have the following characterization of homotopy equivalences in $\mathscr{Q}(\mathscr{C})$. 
(4.4) Proposition. The 1-morphism in $\mathscr{Q}(\mathscr{C})$ given by (4.1) is a homotopy equivalence in $\mathscr{Q}(\mathscr{C})$ if and only if each of the 1-morphisms $\alpha, c, \beta$ and $x$ is a homotopy equivalence in $\mathscr{C}$ and each of the 2-morphisms $L, I, K$ and $J$ is invertible in $\mathscr{C}$.

(4.5) Theorem. Let $\mathscr{C}$ be a 2-category with zeros and suppose that (4.1) specifies a 1-morphism in $\mathscr{Q}(\mathscr{C})$ which has the additional property that $L, I, K$ and $J$ are invertible 2-morphisms in $\mathscr{C}$.

(a) If $w: W \rightarrow C$ is a 1-morphism in $\mathscr{C}$ then $x \circ \sigma(S, w) \subset \sigma\left(S^{\prime}, c w\right)$.

(b) If $x^{\prime}: X^{\prime} \rightarrow Y$ is a 1-morphism in $\mathscr{E}$ then $\sigma\left(x^{\prime}, S^{\prime}\right) \circ c \subset \sigma\left(x^{\prime} x, S\right)$.

Proof. We only prove part (a); the proof of part (b) is similar. We will show by direct verification that $x \circ \sigma(S, w) \subset \sigma\left(S^{\prime}, c w\right)$. Let $\theta=-b K+S w+a H$ be a fixed but arbitrary element of $\sigma(S, w)$. Here $H: o \Rightarrow f w$ and $K: o \Rightarrow g w$ denote invertible 2-morphisms of $\mathscr{C}$. Because $I$ and $L$ are assumed to be invertible, the coherence equation (4.2) can be written

$$
x S=-I g-b^{\prime} L+S^{\prime} c+a^{\prime} K+J f .
$$

Therefore we have:

$$
\begin{aligned}
x \circ \theta & =-x b K+x S w+x a H \\
& =-x b K+\left(-I g-b^{\prime} L+S^{\prime} c+a^{\prime} K+J f\right) \circ w+x a H \\
& =-x b K-I g w-b^{\prime} L w+S^{\prime} c w+a^{\prime} K w+J f w+x a H \\
& =-\left(b^{\prime} L w+I g w+x b K\right)+S^{\prime} c w+\left(a^{\prime} K w+J f w+x a H\right)
\end{aligned}
$$

Noting that the composite 2-morphisms

$$
\begin{aligned}
b^{\prime} L w+I g w+x b K: o & \Rightarrow b^{\prime} g^{\prime} c w \\
a^{\prime} K w+J f w+x a H: o & \Rightarrow a^{\prime} f^{\prime} c w
\end{aligned}
$$

are invertible we thus see that $x \circ \theta$ has been represented as an element of $\sigma\left(S^{\prime}, c w\right)$, as required.

\section{MATRIX TODA BRACKETS}

In this section and in the remainder of the paper $\mathscr{C}$ denotes a fixed 2-category with zeros.

(5.1) Definition. Suppose 1-morphisms in $\mathscr{C}$ are given as indicated below.

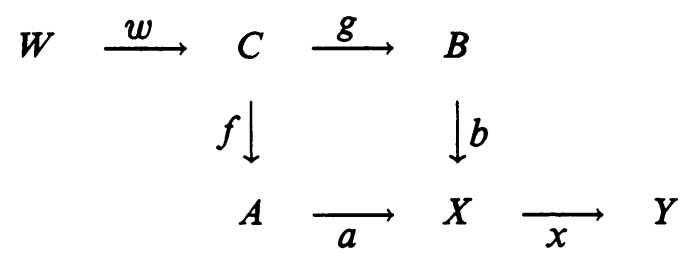

Let $\mathscr{S}=\{S \mid S$ is an invertible 2-morphism $a f \Rightarrow b g\}$. Then we define matrix Toda brackets as follows.

$$
\begin{aligned}
& \left.\left\{\begin{array}{ll}
b, g & , w\} \\
a & f
\end{array}\right\} \bigcup \bigcup \sigma(S, w) \mid S \in \underline{\mathscr{S}}\right\} \\
& \left\{x, \begin{array}{ll}
b, & g \\
a & f
\end{array}\right\}=\bigcup\{\sigma(x, S) \mid S \in \underline{\mathscr{S}}\} .
\end{aligned}
$$


The first operation is nonvacuous (that is, defined) if and only if $f w \simeq 0, g w \simeq$ $o$ and $a f \simeq b g$, and if so is a subset of the group $\mathrm{N}\left(1_{a f w}\right)=\mathrm{N}\left(1_{b g w}\right)$. The second operation is nonvacuous (that is, defined) if and only if $x a \simeq 0, x b \simeq 0$ and $a f \simeq b g$, and if so is a subset of the group $\mathrm{N}\left(1_{x a f}\right)=\mathrm{N}\left(1_{x b g}\right)$.

(5.2) Remark. It follows from Proposition 3.2 that the equations

$$
\left\{\begin{array}{l}
a, f \\
b
\end{array}, w\right\}=-\left\{\begin{array}{ll}
b & g \\
a & f
\end{array}, w\right\}
$$

and

$$
\left\{x, \begin{array}{ll}
a & f \\
b & g
\end{array}\right\}=-\left\{x, \begin{array}{ll}
b, & g \\
a & f
\end{array}\right\}
$$

are valid.

(5.3) Proposition. A matrix Toda bracket depends only on the homotopy classes of the 1-morphisms it contains. That is, in Definition 5.1 suppose that

$$
f \simeq \tilde{f}, \quad g \simeq \tilde{g}, \quad a \simeq \tilde{a}, \quad b \simeq \tilde{b}, \quad w \simeq \tilde{w}, \quad x \simeq \tilde{x} .
$$

Then

$$
\left\{\begin{array}{ll}
b & g \\
a & f
\end{array}, w\right\}=\left\{\begin{array}{ll}
\tilde{b} & \tilde{g} \\
\tilde{a} & \tilde{f}
\end{array}, \tilde{w}\right\}
$$

and

$$
\left\{x, \begin{array}{ll}
b, & g \\
a & f
\end{array}\right\}=\left\{\begin{array}{lll}
\tilde{x}, & \tilde{b} \\
\tilde{a} & \tilde{g} \\
\tilde{f}
\end{array}\right\} .
$$

Proof. It follows directly from Proposition 3.3 that the matrix Toda brackets in Definition 5.1 depend only on the homotopy classes of $w$ and $x$. Now to show their dependence on the homotopy class, say, of $g$, let $H: g \Rightarrow \tilde{g}$ be an invertible 2-morphism. Let $S: a f \Rightarrow b g$ be any invertible 2-morphism. Define $M=b H+S$. Then the two composite 2-morphisms below are equal.
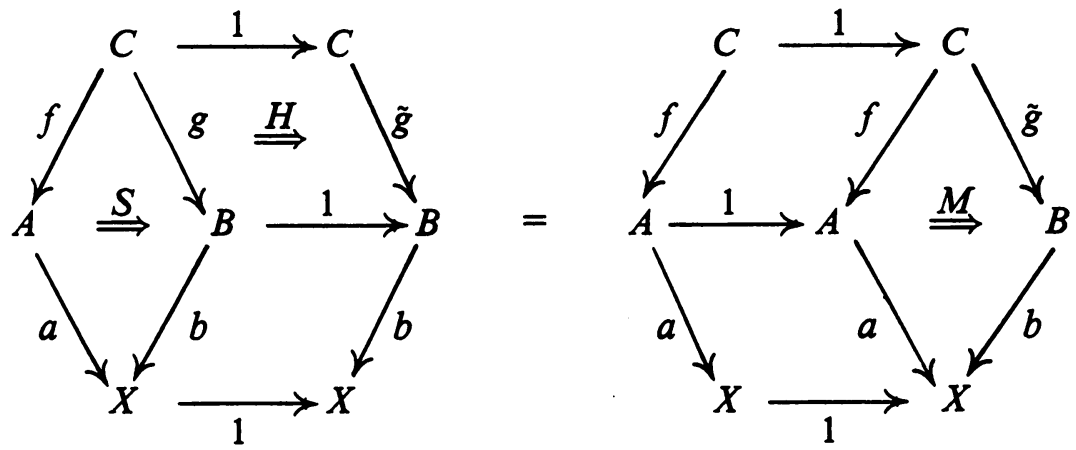

$$
b H+1_{b} g+S=b H+S=M=M+a 1_{f}+1 a f
$$


Consequently the corresponding cube

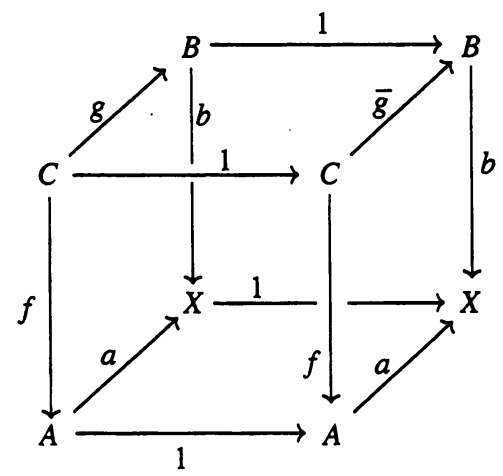

is coherent and so defines a 1 -morphism in $\mathscr{Q}(\mathscr{C})$ which is by Proposition 4.4 a homotopy equivalence in $\mathscr{Q}(\mathscr{C})$. Then by Proposition 4.5(a) we have

$$
\sigma(S, w)=1_{X} \circ \sigma(S, w) \subset \sigma\left(M, 1_{C} \circ w\right)=\sigma(M, w) \subset\left\{\begin{array}{ll}
b & \tilde{g} \\
a & f
\end{array}\right\} .
$$

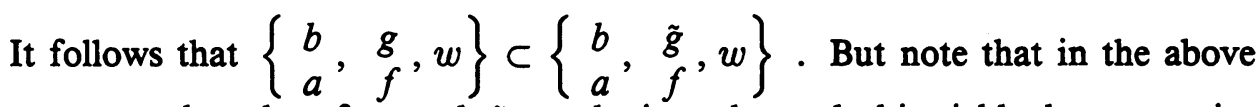
argument the roles of $g$ and $\tilde{g}$ can be interchanged; this yields the reverse inclusion and therefore $\left\{\begin{array}{ll}b, & g \\ a & f\end{array}, w\right\}=\left\{\begin{array}{ll}b, & \tilde{g} \\ a & f\end{array}\right\}$. In similar fashion the dependence on the homotopy classes of $f, a$ and $b$ is shown. This establishes the proposition.

(5.4) Theorem. Let 1-morphisms be given as in Definition 5.1. Then the relations

$$
x \circ\left\{\begin{array}{ll}
b & g \\
a & f
\end{array}, w\right\} \subset\left\{\begin{array}{ll}
x b \\
x a
\end{array}, \begin{array}{l}
g \\
f
\end{array}, w\right\}
$$

and

$$
\left\{x, \begin{array}{ll}
b, & g \\
a
\end{array}\right\} \circ w \subset\left\{x, \begin{array}{ll}
b, & g w \\
a & f w
\end{array}\right\}
$$

are valid. Moreover, if $x$ is a homotopy equivalence in $\mathscr{C}$ then the first inclusion is an equality, and if $w$ is a homotopy equivalence in $\mathscr{C}$ then the second inclusion is an equality.

Proof. We only verify the statements in regard to the first inclusion; those for the second inclusion are similarly verified. So let $S: a f \Rightarrow b g$ be any invertible 2-morphism. Then we must show

$$
x \circ \sigma(S, w) \subset\left\{\begin{array}{ll}
x b \\
x a
\end{array}, \begin{array}{l}
g \\
f
\end{array}, w\right\} .
$$


Note that the coherent cube

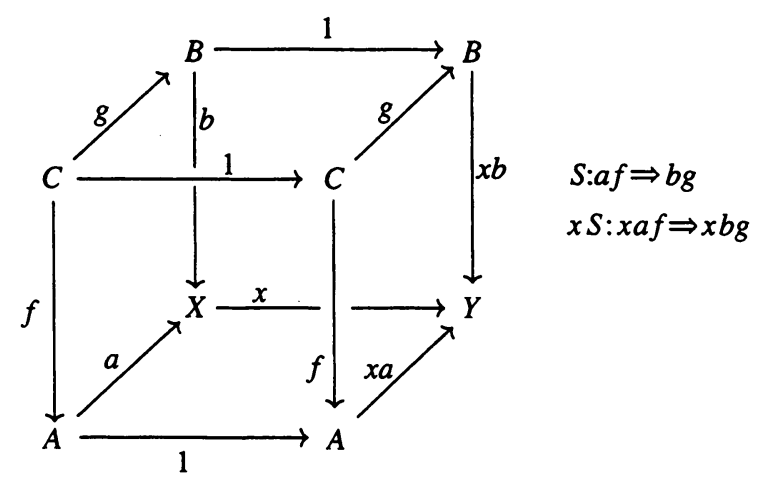

defines a 1 -morphism in $\mathscr{Q}(\mathscr{C})$. Hence by Theorem $4.5(a)$ we have

as required.

$$
x \circ \sigma(S, w) \subset \sigma\left(x S, 1_{C} \circ w\right)=\sigma(x S, w) \subset\left\{\begin{array}{ll}
x b & g \\
x a & f
\end{array}, w\right\},
$$

Now suppose that $x: X \rightarrow Y$ is moreover a homotopy equivalence, with homotopy inverse $\bar{x}: Y \rightarrow X$. By Vogt's Lemma we may choose 2-morphisms $U: \bar{x} x \Rightarrow 1_{X}$ and $V: x \bar{x} \Rightarrow 1_{Y}$ which satisfy $x U=V x$ and $U \bar{x}=\bar{x} V$. Let $T: x a f \Rightarrow x b g$ be an arbitrary invertible 2-morphism. Define $\widetilde{T}: a f \Rightarrow b g$ to be the composite 2-morphism:

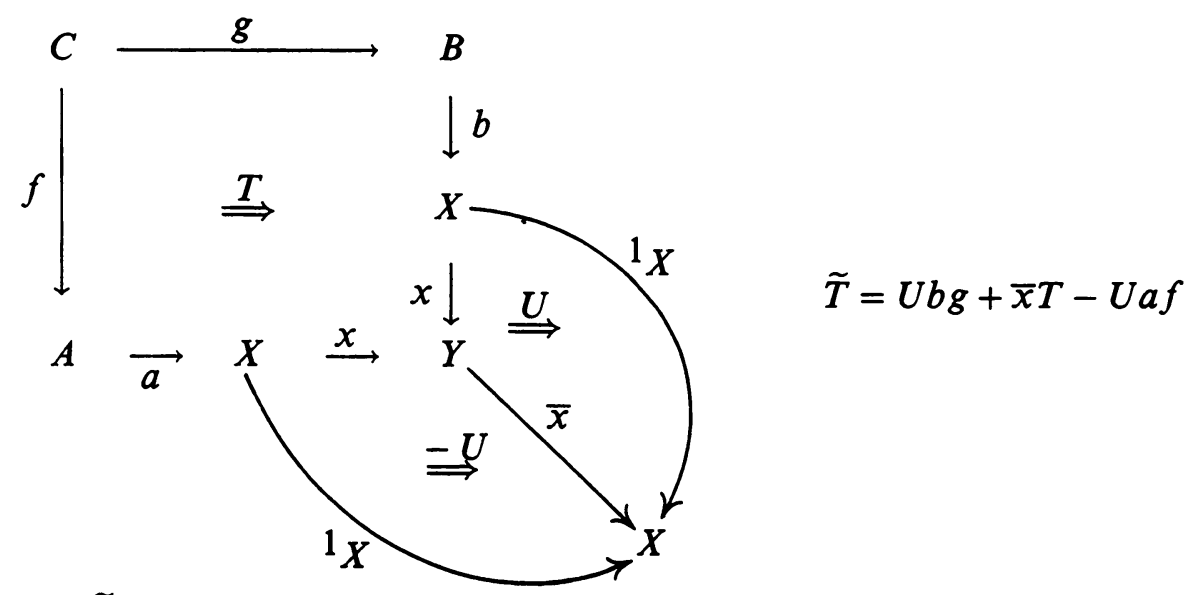

Then $\tilde{T}$ is clearly invertible and

$$
\begin{aligned}
x \widetilde{T} & =x(U g b+\bar{x} T-U a f), & & \text { by definition of } \tilde{T} \\
& =x U b g+x \bar{x} T-x U a f, & & \text { by the interchange law } \\
& =V x b g+x \bar{x} T-V x a f,, & & \text { since } x U=V x \\
& =(V+1 x \bar{x}-V) T, & & \text { by the interchange law } \\
& =T, & & \text { since } V+1_{x \bar{x}}-V=1_{1_{Y}} .
\end{aligned}
$$

From this it follows immediately that

$$
\sigma(T, w) \subset x \circ \sigma(\tilde{T}, w) \subset x \circ\left\{\begin{array}{l}
b, g \\
a
\end{array}, w\right\} .
$$


This shows that $\left\{\begin{array}{ll}x b \\ x a\end{array}, \begin{array}{l}g \\ f\end{array}, w\right\} \subset x \circ\left\{\begin{array}{ll}b \\ a\end{array}, \begin{array}{l}g \\ f\end{array}, w\right\}$ and consequently these two sets are equal.

(5.5) Theorem. Consider a diagram of 1-morphisms as indicated:

$$
\begin{aligned}
& Z \stackrel{\delta}{\longrightarrow} W \stackrel{w}{\longrightarrow} C \stackrel{g}{\longrightarrow} B \\
& f \downarrow \quad \downarrow b \\
& A \quad \underset{a}{\longrightarrow} \underset{x}{\longrightarrow} \quad \boldsymbol{Y} \underset{\gamma}{\longrightarrow} U
\end{aligned}
$$

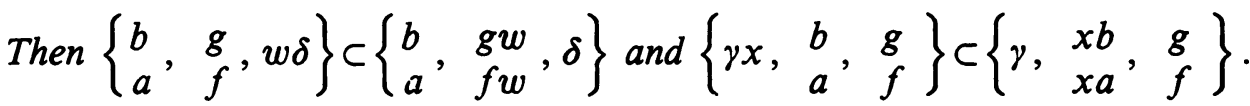
Proof. In order to verify the first inclusion, let $S: a f \Rightarrow b g$ be any invertible 2-morphism. Then we must show $\sigma(S, w \delta) \subset\left\{\begin{array}{ll}b, & g w \\ a & f w\end{array}, \delta\right\}$. Consider the two diagrams:

$$
\begin{aligned}
& Z \stackrel{w \delta}{\longrightarrow} C \stackrel{g}{\longrightarrow} \quad B \quad Z \quad \stackrel{\delta}{\longrightarrow} W \stackrel{g w}{\longrightarrow} B \\
& f \downarrow \stackrel{S}{\Longrightarrow} \downarrow b \quad f w \downarrow \stackrel{S w}{\Longrightarrow} \downarrow b \\
& A \underset{a}{\longrightarrow} X \quad A \quad \underset{a}{\longrightarrow} X
\end{aligned}
$$

Since plainly $\sigma(S w, \delta) \subset\left\{\begin{array}{ll}b, & g w \\ a & f w\end{array}, \delta\right\}$ it suffices to show $\sigma(S, w \delta) \subset$ $\sigma(S w, \delta)$. Actually we claim $\sigma(S, w \delta)=\sigma(S w, \delta)$ in $\mathrm{N}(S w \delta)$. Now an element of $\sigma(S, w \delta)$ is of the form $-b K+S(w \delta)+a H$ where $H: 0 \Rightarrow f w \delta$ and $K: 0 \Rightarrow g w \delta$ are invertible 2-morphisms. But viewed as $-b K+(S w) \delta+a H$ this element also belongs to $\sigma(S w, \delta)$. Thus $\sigma(S, w \delta) \subset \sigma(S w, \delta)$. For the reverse inclusion note that we have the following coherent cube.

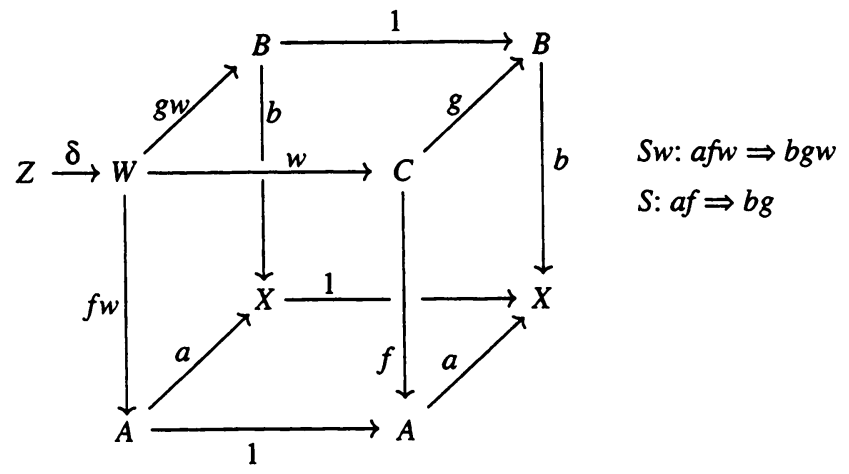


Hence by Theorem 4.5(a) we obtain $\sigma(S w, \delta)=1_{X} \circ \sigma(S w, \delta) \subset \sigma(S, w \delta)$. Therefore $\sigma(S, w \delta)=\sigma(S w, \delta)$, as claimed. The second inclusion in the statement of the proposition is proven in a similar way.

\section{ADMISSIBILITY AND RELATIONS BETWEEN SUBGROUPS}

(6.1) Definition. Let $\{\mathscr{G} ;+\}$ be a group and let $\mathscr{K}_{1}, \ldots, \mathscr{H}_{n}$ be nonempty subsets of $\mathscr{G}$. Define $\mathscr{H}_{1}+\cdots+\mathscr{H}_{n}=\left\{x_{1}+\cdots+x_{n} \mid x_{i} \in \mathscr{H}_{i}, i=1, \ldots, n\right\}$. Note that this is just a subset of $\mathscr{G}$. Of course it is always true that

$$
\mathscr{X}_{1}+\cdots+\mathscr{H}_{n} \subset\left[\mathscr{H}_{1} \cup \cdots \cup \mathscr{H}_{n}\right]
$$

where the latter denotes the subgroup of $\mathscr{G}$ generated by $\mathscr{H}_{1} \cup \ldots \cup \mathscr{K}_{n}$.

The proof of the following elementary result is omitted.

(6.2) Proposition. Suppose that $\mathscr{H}_{1}$ and $\mathscr{H}_{2}$ are subgroups of a group $\mathscr{G}$. Then the following statements are equivalent.

(a) $\mathscr{H}_{1}+\mathscr{H}_{2}$ is a subgroup of $\mathscr{G}$.

(b) $\mathscr{H}_{1}+\mathscr{H}_{2}=\mathscr{H}_{2}+\mathscr{H}_{1}$.

(c) $\mathscr{K}_{1}+\mathscr{H}_{2}=\left[\mathscr{K}_{1} \cup \mathscr{H}_{2}\right]$.

Definition. Let 1-morphisms in $\mathscr{C}$

$$
W \stackrel{w}{\longrightarrow} C \stackrel{f}{\longrightarrow} A \stackrel{a}{\longrightarrow} X
$$

be given. Then the functions

$$
\begin{array}{ll}
\mathscr{A}(f) \longrightarrow \mathscr{A}(a f), & \xi \mapsto a \xi, \\
\mathscr{A}(f) \longrightarrow \mathscr{A}(f w), & \xi \mapsto \xi w,
\end{array}
$$

where $\xi \in \mathscr{A}(f)$, are group homomorphisms. The corresponding images of a subset $\mathscr{E} \subset \mathscr{A}(f)$ under these homomorphisms will be denoted respectively by $a \circ \mathscr{E}$ in $\mathscr{A}(a f)$ and by $\mathscr{E} \circ w$ in $\mathscr{A}(f w)$. Of course these images are subgroups if $\mathscr{E}$ is a subgroup. We will say that the above composite af $w$ is admissible if $\mathscr{A}(a f) \circ w+a \circ \mathscr{A}(f w)$ is a subgroup of $\mathscr{A}(a f w)$. Note that if $\mathscr{A}(a f w)$ is abelian then $a f w$ is admissible.

Now if $a f w$ is admissible then by Proposition 6.2 we have:

$$
\mathscr{A}(a f) \circ w+a \circ \mathscr{A}(f w)=a \circ \mathscr{A}(f w)+\mathscr{A}(a f) \circ w
$$

Diagramatically this means that a diagram of the form on the left below can be replaced by a diagram of the form on the right below, and vice versa.
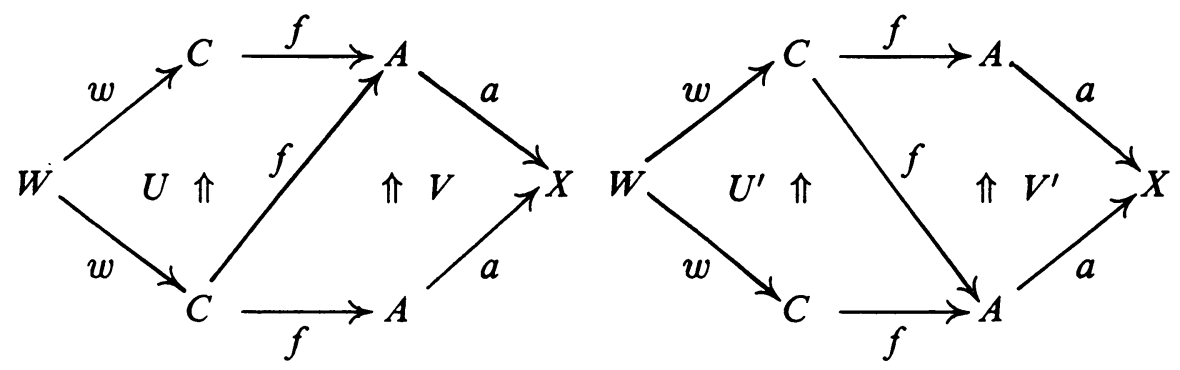
That is, $a U+V w=V^{\prime} w+a U^{\prime}$. Here $U, U^{\prime}, V, V^{\prime}$ are to be invertible 2-morphisms.

(6.4) Proposition. (a) If $T: g \Rightarrow f$ is an invertible 2-morphism then the function

$$
\left(\mathrm{)}: \mathscr{A}(f) \longrightarrow \mathscr{A}(g), \quad \xi \mapsto \xi^{T}=-T+\xi+T,\right.
$$

is an isomorphism of groups. Furthermore if $\mathscr{A}(f)$ is abelian then the isomorphism ( $)^{T}$ is independent of the specific invertible 2-morphism $T$ used to define it.

(b) Let $f: C \rightarrow A$ satisfy $f \simeq 0$. Then $\mathscr{A}(f)=\left\{\xi: f \Rightarrow f \mid \xi\langle\right.$ conj $\left.\rangle 1_{o}\right\}$.

Proof. (a) That ( ) $T$ is a group isomorphism is easily verified and so omitted. Now let also $T^{\prime}: g \Rightarrow f$ be an invertible 2-morphism. Then $\left(T^{\prime}-T\right): f \Rightarrow f$ and $\left(T-T^{\prime}\right): f \Rightarrow f$ are invertible and so both belong to $\mathscr{A}(f)$. Let $\xi \in \mathscr{A}(f)$. Then because $\mathscr{A}(f)$ is assumed to be abelian we have:

$$
\begin{aligned}
\xi^{T^{\prime}} & =-T^{\prime}+\xi+T^{\prime} \\
& =-T+\left(T-T^{\prime}\right)+\xi+\left(T^{\prime}-T\right)+T \\
& =-T+\xi+\left(T-T^{\prime}\right)+\left(T^{\prime}-T\right)+T \\
& =-T+\xi+T \\
& =\xi
\end{aligned}
$$

This show the nondependence of ( $)^{T}$ on $T$.

(b) Since $f \simeq 0$, we have ${ }^{\prime} f$ con $\left.j\right\rangle 1_{O}$. Hence a 2-morphism $\xi: f \Rightarrow f$ is conjugate to $1_{f}$ if and only if $\xi$ is conjugate to $1 o$. The result now follows from Proposition 2.4.

(6.5) Notation. The image of a subset $\mathscr{E} \subset \mathscr{A}(f)$ under the isomorphism ( $)^{T}$ of Proposition 6.1.a will be denoted $\mathscr{E} T$; that is, $\mathscr{E} T=\{-T+\xi+T \mid \xi \in \mathscr{E}\}$. Of course if $\mathscr{E}$ is a subgroup of $\mathscr{A}(f)$ then $\mathscr{E}^{T}$ is a subgroup of $\mathscr{A}(g)$. Also note that $\mathscr{A}(f)^{T}=\mathscr{A}(g)$.

The proof of the following proposition, being straightforward, is omitted.

(6.6) Proposition. (a) Let 1-morphisms $w: W \rightarrow C, f_{0}, f_{1}: C \rightarrow A$ and a: $A \rightarrow X$ in $\mathscr{C}$ be given. Also suppose there is given an invertible 2-morphism $T: f_{1} \Rightarrow f_{0}$. Then for any subset $\mathscr{E} \subset \mathscr{A}\left(f_{0}\right)$ the equations $a \circ \mathscr{E} T=(a \circ \mathscr{E})^{a T}$ in $\mathscr{A}\left(a f_{1}\right)$ and $\mathscr{E}^{T} \circ w=(\mathscr{E} \circ w)^{T} w$ in $\mathscr{A}\left(f_{1} w\right)$ hold.

(b) Let $S: h \Rightarrow g$ and $T: g \Rightarrow f$ be invertible 2-morphisms and $\mathscr{E} \subset \mathscr{A}(g)$. Then $\mathscr{E}^{S}=(\mathscr{E}-T) T+S$ in $\mathscr{A}(h)$.

(6.7) Proposition. For 1-morphisms $W \stackrel{w}{\longrightarrow} C \stackrel{f}{\longrightarrow} A$ in $\mathscr{C}$ let $L: o \Rightarrow f w$ be an invertible 2-morphism. Then the group $\mathscr{A}(f w)$ is abelian if and only if the group $\mathrm{N}\left(1_{f w}\right)=\mathrm{N}\left(1_{o}\right)=\mathscr{A}(o: W \rightarrow A)$ is abelian. And when this is assumed to be the case we further have:

(a) If $f \simeq o$ then $(\mathscr{A}(f) \circ w)^{L}=\mathscr{A}(o: C \rightarrow A) \circ w$.

(b) If $w \simeq o$ then $(f \circ \mathscr{A}(w))^{L}=f \circ \mathscr{A}(o: W \rightarrow C) \circ w$. 
Proof. The first assertion is clear since ()$^{L}: \mathscr{A}(f w) \longrightarrow \mathscr{A}(0: W \rightarrow A)$ is an isomorphism. Now since the proofs of parts (a) and (b) are similar we shall give only the proof for (a). So let $\xi \in \mathscr{A}(f)$. Since $f \simeq 0$ we have $\xi\left\langle\right.$ conj $j 1_{0}$ by Proposition 6.1.b. Hence there exists invertible 2-morphisms $U: f \Rightarrow 0$ and $V: o \Rightarrow f$ such that $\xi=V+1 o+U=V+U$. Then

$$
\begin{aligned}
-L+\xi w+L & =-L+(V+U) w+L \\
& =(-L+V w)+(U w+L) \\
& =(U w+L)+(-L+V w), \quad \text { since } \mathscr{A}(o: W \rightarrow A) \text { is abelian } \\
& =U w+V w \\
& =(U+V) w \\
& \in \mathscr{A}(o: C \rightarrow A) \circ w .
\end{aligned}
$$

Conversely, let $\chi \in \mathscr{A}(o: C \rightarrow A)$. Then $\chi\langle c o n j\rangle 1_{o}\langle c o n j\rangle 1_{f}$, the last relation obtaining because $f \simeq 0$. Hence there exist invertible 2-morphisms $R: 0 \Rightarrow f$ and $T: f \Rightarrow 0$ such that $\chi=T+1_{f}+R=T+R$. Then

$$
\begin{aligned}
\chi w & =(T+R) w \\
& =T w+R w \\
& =(T w+L)+(-L+R w) \\
& =(-L+R w)+(T w+L), \quad \text { since } \mathscr{A}(f w) \text { is abelian } \\
& =-L+(R+T) w+L \\
& \in(\mathscr{A}(f) \circ w)^{L} .
\end{aligned}
$$

Hence $(\mathscr{A}(f) \circ w)^{L}=\mathscr{A}(o: C \rightarrow A) \circ w$.

(6.8) Proposition. Suppose the composite af $w$ is as in Definition 6.3.

(a) Let $H: 0 \Rightarrow f w$ be a given invertible 2-morphism. Then

$$
(\mathscr{A}(a f) \circ w+a \circ \mathscr{A}(f w))^{a H}=(\mathscr{A}(a f) \circ w)^{a H}+a \circ \mathrm{N}(1 f w)
$$

and consequently if af $w$ is admissible then $(\mathscr{A}(a f) \circ w)^{a H}+a \circ \mathrm{N}\left(1_{f w}\right)$ is a subgroup of $\mathrm{N}\left(1_{a f w}\right)$.

(b) Let $Q: 0 \Rightarrow$ af be a given invertible 2-morphism. Then

$$
(\mathscr{A}(a f) \circ w+a \circ \mathscr{A}(f w))^{Q w}=\mathrm{N}\left(1_{a f}\right) \circ w+(a \circ \mathscr{A}(f w))^{Q w}
$$

and consequently if afw is admissible then $\mathrm{N}\left(1_{a f}\right) \circ w+(a \circ \mathscr{A}(f w)) Q w$ is a subgroup of $\mathrm{N}\left(1_{a f} w\right)$.

Proof. (a) Under the isomorphism

$$
()^{a H}: \mathscr{A}(a f w) \longrightarrow \mathscr{A}(o: W \rightarrow X)=\mathrm{N}\left(1_{a f w}\right)
$$

we have

$$
\left((\mathscr{A}(a f) \circ w+a \circ \mathscr{A}(f w))^{a H}=(\mathscr{A}(a f) \circ w)^{a H}+(a \circ \mathscr{A}(f w))^{a H} .\right.
$$

Also 


$$
\begin{aligned}
(a \circ \mathscr{A}(f w))^{a H} & =a \circ(\mathscr{A}(f w))^{H} & & \text {, by Proposition 6.6.a } \\
& =a \circ \mathscr{A}(0: W \rightarrow A) & & \\
& =a \circ \mathrm{N}\left(1_{0}\right) & & \text {, by Proposition 2.4 } \\
& =a \circ \mathrm{N}\left(1_{f w}\right) & & \text {, by Proposition 2.2.d. }
\end{aligned}
$$

This establishes part (a).

(b) The proof of (b) is similar to that for (a) and hence omitted.

(6.9) Proposition. With reference to the diagram given in Definition 5.1 suppose that there exist invertible 2-morphisms $H: 0 \Rightarrow f w, K: 0 \Rightarrow g w$ and $S: a f \Rightarrow b g$. Let $\mathscr{N}=\mathrm{N}\left(1_{a f w}\right)=\mathrm{N}\left(1_{b g w}\right)=\mathscr{A}(o: W \rightarrow X)$. Then:

(a) If any one of the three groups $\mathscr{A}(a f w), \mathscr{A}(b g w)$ or $\mathscr{N}$ is abelian then so are the other two.

(b) Suppose that $\mathscr{N}$ (or equivalently by part (a) either $\mathscr{A}($ a fw) or $\mathscr{A}(b g w)$ ) is assumed to be abelian then

$$
(\mathscr{A}(a f) \circ w)^{a H}=(\mathscr{A}(b g) \circ w)^{b K}
$$

as subgroups of the group $\mathscr{N}$. Let this subgroup be denoted by $\mathscr{D}$, say. Then furthermore $\mathscr{D}$ is independent of the specific invertible 2-morphisms $H$ and $K$ used to define it.

(c) In part (b), if $\mathrm{N}(S)$ is nonvacuous then also $\mathscr{D}=\mathrm{N}(S) \circ w$.

Proof. (a) This is clear since there exist isomorphisms

$$
\mathscr{A}(b g w) \stackrel{()^{-S w}}{\longrightarrow} \mathscr{A}(a f w) \stackrel{()^{a H}}{\longrightarrow} \mathscr{A}(o: W \rightarrow X)
$$

(b) First observe that

$$
\begin{aligned}
(\mathscr{A}(a f) \circ w)^{a H} & =\left((\mathscr{A}(a f) \circ w)^{-S w}\right)^{S w+a H} & & , \text { by Proposition 6.6.b } \\
& =\left(\mathscr{A}(a f)^{-S} \circ w\right)^{S w+a H} & & , \text { by Proposition 6.6.a } \\
& =(\mathscr{A}(b g) \circ w)^{S w+a H} & & \text {, since } \mathscr{A}(a f)^{-S}=\mathscr{A}(b g) .
\end{aligned}
$$

Also since $S w+a H$ and $b K$ are both invertible 2-morphisms $0 \Rightarrow b g w$ and since by hypothesis $\mathscr{A}(b g w)$ is abelian we may use Proposition 6.4.a to deduce that

$$
(\mathscr{A}(b g) \circ w)^{S w+a H}=(\mathscr{A}(b g) \circ w)^{b K} .
$$

The stated equality of the two subgroups is now obtained. The last statement of part (b) clearly follows by using Proposition 6.4.a.

(c) By Proposition 2.5 we have $\mathrm{N}(S)=\mathscr{A}(0: C \rightarrow X)$ because $\mathrm{N}(S)$ is assumed nonvacuous. Of course this is so if and only if $a f \simeq 0$ and $b g \simeq 0$. Now since $a f \simeq 0$ and $\mathscr{N}$ is abelian we may apply Proposition 6.7.a to deduce

$$
\mathscr{D}=(\mathscr{A}(a f) \circ w)^{a H}=\mathscr{A}(0: C \rightarrow X) \circ w=\mathrm{N}(S) \circ w
$$

as claimed.

The proof of the following proposition is omitted; it is analogous to the proof of Proposition 6.9. 
(6.10) Proposition. With reference to the diagram given in Definition 5.1 suppose that there exist invertible 2-morphisms $U: 0 \Rightarrow x a, V: 0 \Rightarrow x b$ and $S: a f \Rightarrow b g$. Let $\mathscr{H}=\mathrm{N}\left(1_{x a f}\right)=\mathrm{N}\left(1_{x b g}\right)=\mathscr{A}(o: C \rightarrow Y)$. Then:

(a) If any one of the three groups $\mathscr{A}(x a f), \mathscr{A}(x b g)$ or $\mathscr{M}$ is abelian then so are the other two.

(b) Suppose that $\mathscr{M}$ (or equivalently by part (a) either $\mathscr{A}(x a f)$ or $\mathscr{A}(x b g)$ ) is assumed to be abelian then

$$
(x \circ \mathscr{A}(a f))^{U f}=(x \circ \mathscr{A}(b g))^{V g}
$$

as subgroups of the group $\mathscr{M}$. Let this subgroup be denoted by $\mathscr{R}$, say. Then furthermore $\mathscr{R}$ is independent of the specific invertible 2-morphisms $U$ and $V$ used to define it.

(c) In part (b), if $\mathrm{N}(S)$ is nonvacuous then also $\mathscr{R}=x \circ \mathrm{N}(S)$.

\section{INDETERMINACIES OF MATRIX TODA BRACKETS}

For convenience of reference we repeat the diagram in Definition 5.1:

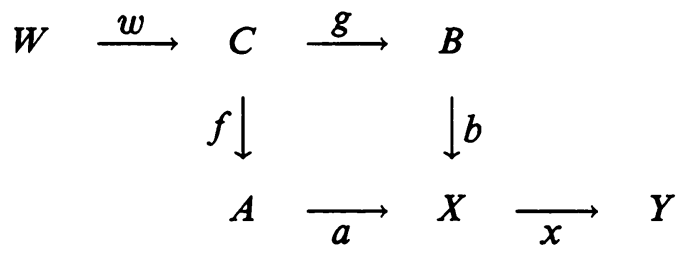

Suppose now that invertible 2-morphisms $H: 0 \Rightarrow f w, K: 0 \Rightarrow g w, S: a f \Rightarrow$ $b g, U: 0 \Rightarrow x a$ and $V: o \Rightarrow x b$ exist. Then both matrix Toda brackets $\left\{\begin{array}{ll}b & g \\ a & f\end{array}, w\right\}$ and $\left\{x, \begin{array}{ll}b \\ a\end{array}, \begin{array}{l}g \\ f\end{array}\right\}$ are defined. Let $\theta=-b K+S w+a H \in$ $\mathscr{A}(o: W \rightarrow X)$ and $\phi=-V g+x S+U f \in \mathscr{A}(0: C \rightarrow Y)$. Also set

$$
\mathscr{N}=\mathrm{N}\left(1_{a f w}\right)=\mathrm{N}\left(1_{b g w}\right)=\mathscr{A}(o: W \rightarrow X)
$$

and

$$
\mathscr{M}=\mathrm{N}\left(1_{x a f}\right)=\mathrm{N}\left(1_{x b g}\right)=\mathscr{A}(o: C \rightarrow Y) .
$$

Throughout this section we will maintain the notations and assumptions just introduced.

(7.1) Theorem. Let notation be as introduced above. Then we have:
(a) $\left\{\begin{array}{ll}b & g \\ a & f\end{array}, w\right\}=b \circ \mathrm{N}(1 g w)+\theta+(\mathscr{A}(a f) \circ w)^{a H}+a \circ \mathrm{N}(1 f w)$.
(b) $\left\{\begin{array}{ll}b & g \\ a & f\end{array}\right\}=b \circ \mathrm{N}(1 g w)+(\mathscr{A}(b g) \circ w)^{b K}+\theta+a \circ \mathrm{N}(1 f w)$.
(c) $\left\{x, \begin{array}{ll}b, & g \\ a\end{array}\right\}=\mathrm{N}\left(1_{x b}\right) \circ g+\phi+(x \circ \mathscr{A}(a f)) U f+\mathrm{N}(1 x a) \circ f$.
(d) $\left\{x, \begin{array}{ll}b, & g \\ a & f\end{array}\right\}=\mathrm{N}\left(1_{x b}\right) \circ g+(x \circ \mathscr{A}(b g))^{V b}+\phi+\mathrm{N}\left(1_{x a}\right) \circ f$.

The right-hand sums of these equations are subsets in the sense of Definition 6.1 . 
Proof. (a) Set $\mathscr{K}=b \circ \mathrm{N}(1 g w)+\theta+(\mathscr{A}(a f) \circ w+a \circ \mathscr{A}(f w))^{a H}$. By Proposition 6.8.a it will be sufficient to verify that $\left\{\begin{array}{l}b, g \\ a\end{array}, w\right\}=\mathscr{K}$. First suppose that $-b K^{\prime}+S^{\prime} w+a H^{\prime}$ is an arbitrary element of $\left\{\begin{array}{l}b, g \\ a\end{array}, w\right\}$ where $H^{\prime}: o \Rightarrow f w, K^{\prime}: o \Rightarrow g w$ and $S^{\prime}: a f \Rightarrow b g$ are invertible 2-morphisms. Then

$$
\begin{aligned}
-b K^{\prime} & +S^{\prime} w+a H^{\prime} \\
& =-b K^{\prime}+b K-b K+S w+a H-a H-S w+S^{\prime} w+a H^{\prime}-a H+a H \\
& =b\left(-K^{\prime}+K\right)+\theta+\left(-S w+S^{\prime} w+a H^{\prime}-a H\right)^{a H} \\
& =b\left(-K^{\prime}+K\right)+\theta+\left(\left(-S+S^{\prime}\right) w+a\left(H^{\prime}-H\right)\right)^{a H} .
\end{aligned}
$$

Note that $-K^{\prime}+K \in \mathrm{N}(1 g w), \quad-S+S^{\prime} \in \mathscr{A}(a f), \quad H^{\prime}-H \in \mathscr{A}(f w)$ so that $b\left(-K^{\prime}+K\right)+\theta+\left(\left(-S+S^{\prime}\right) w+a\left(H^{\prime}-H\right)\right)^{a H} \in \mathscr{K}$.

Next we show that $\mathscr{K} \subset\left\{\begin{array}{l}b, g \\ a\end{array}, \begin{array}{l}f \\ w\end{array}\right\}$. An arbitrary element of $\mathscr{K}$ is of the form $b L+\theta+\xi^{a H}$ where $L: o \Rightarrow o: W \rightarrow B$ belongs to $\mathrm{N}(1 g w)$ and $\xi$ is an element of $\mathscr{A}(a f) \circ w+a \circ \mathscr{A}(f w)$. We may write $\xi=V w+a U$ for some $V \in \mathscr{A}(a f)$ and some $U \in \mathscr{A}(f w)$. Then

$$
\begin{aligned}
b L+\theta+\xi^{a H} & =b L-b K+S w+a H-a H+V w+a U+a H \\
& =b(L-K)+S w+V w+a(U+H) \\
& =-b(K-L)+(S+V) w+a(U+H) .
\end{aligned}
$$

Observe that $K-L: o \Rightarrow g w, S+V: a f \Rightarrow b g$ and $U+H: o \Rightarrow f w$ so that this last expression is an element of $\left\{\begin{array}{l}b, g \\ a\end{array}, w\right\}$. Therefore we may conclude that $\left\{\begin{array}{l}b \\ a\end{array}, \underset{f}{g}, w\right\}=\mathscr{K}$ as claimed.

(b) We have

$$
\begin{aligned}
\left\{\begin{array}{l}
b \\
a
\end{array}, \begin{array}{l}
g \\
f
\end{array}\right\}=-\left\{\begin{array}{ll}
a & f \\
b & g
\end{array}, w\right\} \quad \text { (by Remark 5.2) } \\
=-\left(a \circ \mathrm{N}(1 f w)+(-a H-S w+b K)+(\mathscr{A}(b g) \circ w)^{b K}+b \circ \mathrm{N}(1 g w)\right)
\end{aligned}
$$

(by use of part (a) above)

$$
=b \circ \mathrm{N}(1 g w)+(\mathscr{A}(b g) \circ w)^{b K}+\theta+a \circ \mathrm{N}(1 f w) \text {, }
$$

where in the last equality we have made use of the fact that $-\mathscr{H}=\mathscr{H}$ whenever $\mathscr{H}$ is a subgroup of a group $\mathscr{G}$. Note that it is also possible to deduce part (b) from part (a) by verifying directly that

$$
\theta+(\mathscr{A}(a f) \circ w)^{a H}=(\mathscr{A}(b g) \circ w)^{b K}+\theta .
$$

(c) and (d). The proofs for these parts are analogous to those just given for parts (a) and (b) respectively and hence are omitted. 
(7.2) Corollary. Let notation be as introduced above. Then we have:

(a) If the composite af $w$ is admissible then $\left\{\begin{array}{l}b \\ a\end{array}, \begin{array}{l}g \\ f\end{array}, w\right\}$ is a double coset of the subgroups $b \circ \mathrm{N}\left(1_{g w}\right)$ and $(\mathscr{A}(a f) \circ w)^{a H}+a \circ \mathrm{N}\left(1_{f w}\right)$ of $\mathscr{N}$.

(b) If the composite $b g w$ is admissible then $\left\{\begin{array}{l}b \\ a\end{array}, \begin{array}{l}g \\ f\end{array}, w\right\}$ is a double coset of the subgroups $b \circ \mathrm{N}(1 g w)+(\mathscr{A}(b g) \circ w)^{b K}$ and $a \circ \mathrm{N}\left(1_{f w}\right)$ of $\mathscr{N}$.

(c) If the composite xaf is admissible then $\left\{x, \begin{array}{ll}b \\ a\end{array}, \begin{array}{l}g \\ f\end{array}\right\}$ is a double coset of the subgroups $\mathrm{N}\left(1_{x b}\right) \circ g$ and $(x \circ \mathscr{A}(a f)) U f+N\left(1_{x a}\right) \circ f$ of $\mathscr{M}$.

(d) If the composite $x b g$ is admissible then $\left\{x, \begin{array}{l}b, g \\ a\end{array}\right\}$ is a double coset of the subgroups $\mathrm{N}\left(1_{x b}\right) \circ g+(x \circ \mathscr{A}(b g)) V g$ and $\mathrm{N}\left(1_{x a}\right) \circ f$ of $\mathscr{M}$.

Proof. Only the proof for part (a) will be given; those for (b)-(c) are similar. By Proposition 6.8.a, $(\mathscr{A}(a f) \circ w)^{a H}+a \circ \mathrm{N}(1 f w)$ is a subgroup of $\mathscr{N}$ since $a f w$ is admissible. Hence the statement in part (a) follows immediately from the equation in Theorem 7.1.a .

(7.3) Corollary. (a) Assume that $\mathscr{N}$ is abelian and let $\mathscr{D}$ be the subgroup of $\mathscr{N}$ identified in Proposition 6.9.b. Then the matrix Toda bracket $\left\{\begin{array}{l}b, g \\ a\end{array}, w\right\}$ is a coset of the subgroup $b \circ \mathrm{N}(1 g w)+\mathscr{D}+a \circ \mathrm{N}\left(1_{f w}\right)$ of $\mathscr{N}$.

(b) Assume that $\mathscr{M}$ is abelian and let $\mathscr{R}$ be the subgroup of $\mathscr{N}$ identified in Proposition 6.10.b. Then the matrix Toda bracket $\left\{x, \begin{array}{l}b \\ a\end{array}, \begin{array}{l}g \\ f\end{array}\right\}$ is a coset of the subgroup $\mathrm{N}\left(1_{x b}\right) \circ g+\mathscr{R}+\mathrm{N}\left(1_{x a}\right) \circ f$ of $\mathscr{M}$.

(c) If $\mathrm{N}(S) \neq \varnothing$ (or equivalently if $a f \simeq 0 \simeq b g$ ) then $\mathscr{D}=\mathrm{N}(S) \circ w$ in (a), and $\mathscr{R}=x \circ \mathrm{N}(S)$ in (b).

Proof. We note that part (c) follows directly from Proposition 6.9.c and Proposition 6.10.c. Of (a) and (b), only the proof for (a) will be given; that for (b) is analogous. First note that $b \circ \mathrm{N}(1 g w)+\mathscr{D}+a \circ \mathrm{N}(1 f w)$ is a subgroup of $\mathscr{N}$ because $\mathscr{N}$ is abelian. Also, by Proposition 6.9.a, $\mathscr{A}(a f w)$ must be abelian and hence the composite $a f w$ is admissible. Then by Theorem 7.1.a and Proposition 6.9.b we have

$$
\begin{aligned}
\left\{\begin{array}{l}
b, g \\
a
\end{array}, w\right\} & =b \circ \mathrm{N}\left(1_{g w}\right)+\theta+(\mathscr{A}(a f) \circ w)^{a H}+a \circ \mathrm{N}\left(1_{f w}\right) \\
& =b \circ \mathrm{N}\left(1_{g w}\right)+\theta+\mathscr{D}+a \circ \mathrm{N}\left(1_{f w}\right) \\
& =\theta+b \circ \mathrm{N}\left(1_{g w}\right)+\mathscr{D}+a \circ \mathrm{N}\left(1_{f w}\right) .
\end{aligned}
$$

That is, $\left\{\begin{array}{ll}b \\ a\end{array}, \begin{array}{l}g \\ f\end{array}\right\}$ is a coset of $b \circ \mathrm{N}\left(1_{g} w\right)+\mathscr{D}+a \circ \mathrm{N}\left(1_{f w}\right)$. 


\section{THE Classical TOda BRACKet}

The case of the classical Toda bracket deserves mention in our context.

(8.1) Definition. Let 1-morphisms in $\mathscr{C}$

$$
W \stackrel{w}{\longrightarrow} C \stackrel{g}{\longrightarrow} B \stackrel{b}{\longrightarrow} X
$$

be given. Following Toda's definition [12, (1.14), p. 9] in the topological case we refer to the subset $\{b, g, w\}$ of $\mathrm{N}(1, g g w)$ given by

$\{b, g, w\}=\{-b K+L w \mid K: o \Rightarrow g w, L: o \Rightarrow b g$ and $K$ and $L$ are invertible $\}$ as the classical Toda bracket. Of course $\{b, g, w\}$ is nonvacuous (that is, defined) if and only if $g w \simeq 0$ and $b g \simeq 0$. Also it is to be noted that what is here designated $\{b, g, w\}$ corresponds to what is designated $-\{b, g, w\}$ in [4].

(8.2) Proposition. Suppose that $K: 0 \Rightarrow g w$ and $L: O \Rightarrow b g$ are fixed invertible 2-morphisms and let $\rho=-b K+L w$. Then $\{b, g, w\}$ is defined and:

(a) $\{b, g, w\}=b \circ \mathrm{N}(1 g w)+\rho+\mathrm{N}\left(1_{b g}\right) \circ w$. That is, $\{b, g, w\}$ is a double coset of the subgroups $b \circ \mathrm{N}(1 g w)$ and $\mathrm{N}\left(1_{b g}\right) \circ w$.

(b) If the composite $b g w$ is admissible then $\{b, g, w\}$ is a coset of the subgroup $(b \circ \mathscr{A}(g w))^{L w}+\mathrm{N}\left(1_{b g}\right) \circ w$.

(c) If the group $\mathrm{N}\left(1_{b g w}\right)$ is abelian then $\{b, g, w\}$ is a coset of the subgroup $b \circ \mathrm{N}\left(1_{g w}\right)+\mathrm{N}\left(1_{b g}\right) \circ w$.

Proof. (a) Let $-b K^{\prime}+L^{\prime} w \in\{b, g, w\}$ be an arbitrary element. Then

$$
\begin{aligned}
-b K^{\prime}+L^{\prime} w & =-b K^{\prime}+b K-b K+L w-L w+L^{\prime} w \\
& =b\left(-K^{\prime}+K\right)+\rho+\left(-L+L^{\prime}\right) w \\
& \in b \circ \mathrm{N}(1 g w)+\rho+\mathrm{N}\left(1_{b g}\right) \circ w,
\end{aligned}
$$

the last relation since $-K^{\prime}+K \in \mathrm{N}(1 g w)$ and $-L+L^{\prime} \in \mathrm{N}\left(1_{b g}\right)$.

Conversely if $\xi \in \mathrm{N}(1 g w)$ and $\chi \in \mathrm{N}(1 b g)$ then we may write $\xi=-K^{\prime}+K^{\prime \prime}$ and $\chi=-L^{\prime \prime}+L^{\prime}$ where $K^{\prime}, K^{\prime \prime}: 0 \Rightarrow g w$ and $L^{\prime}, L^{\prime \prime}: 0 \Rightarrow b g$ are invertible 2-morphisms. Then

$$
\begin{aligned}
b \xi+\rho+\chi w & =b\left(-K^{\prime}+K^{\prime \prime}\right)+(-b K+L w)+\left(-L^{\prime \prime}+L^{\prime}\right) w \\
& =-b\left(K-K^{\prime \prime}+K^{\prime}\right)+\left(L-L^{\prime \prime}+L^{\prime}\right) w \\
& \in\{b, g, w\} .
\end{aligned}
$$

Therefore $\{b, g, w\}=b \circ \mathrm{N}(1 g w)+\rho+\mathrm{N}\left(1_{b g}\right) \circ w$.

(b) A direct verification shows that $b \circ \mathrm{N}(1 g w)+\rho=\rho+(b \circ \mathscr{A}(g w))^{L w}$. Hence from part (a) we have

$$
\{b, g, w\}=\rho+(b \circ \mathscr{A}(g w))^{L w}+\mathrm{N}\left(1_{b g}\right) \circ w .
$$

Now because $b g w$ is assumed to be admissible Proposition 6.8.b implies that the subset $\mathrm{N}\left(1_{b g}\right) \circ w+(b \circ \mathscr{A}(g w))^{L w}$ is a subgroup. But this latter, being 
a subgroup, is the same as $(b \circ \mathscr{A}(g w))^{L w}+\mathrm{N}\left(1_{b g}\right) \circ w$. This completes the proof.

(c) If $\mathrm{N}\left(1_{b g w}\right)$ is abelian then $b \circ \mathrm{N}(1 g w)+\mathrm{N}\left(1_{b g}\right) \circ w$ is a subgroup of $\mathrm{N}\left(1_{b g w}\right)$ and the statement is immediate by part (a).

(8.3) Proposition. (a) If $a: A \rightarrow X$ is any 1-morphism whatsoever then we have

$$
\{b, g, w\} \subset\left\{\begin{array}{ll}
b & g \\
a & o
\end{array}, w\right\} \text { and }-\{b, g, w\} \subset\left\{\begin{array}{ll}
a & o \\
b & g
\end{array}, w\right\} \text {. }
$$

And moreover $\{b, g, w\}=\left\{\begin{array}{ll}b & g \\ 0 & 0\end{array}, w\right\}$.

(b) If $h: W \rightarrow D$ is any 1-morphism whatsoever then we have

$$
\{b, g, w\} \subset\left\{b, \begin{array}{ll}
o \\
g
\end{array}, \begin{array}{l}
h \\
w
\end{array}\right\} \text { and }-\{b, g, w\} \subset\left\{b, \begin{array}{ll}
g & w \\
o & h
\end{array}\right\} \text {. }
$$

And moreover $\{b, g, w\}=\left\{b, \begin{array}{ll}o & 0 \\ g\end{array}\right\}$.

Proof. We only prove part (a); the proof of part (b), being similar, is omitted. Given $K: 0 \Rightarrow g w$ and $L: 0 \Rightarrow b g$, note that:

$$
\begin{aligned}
-b K+L w & =-b K+L w+1(o: W \rightarrow X) \\
& =-b K+L w+a 1(o: W \rightarrow A) \\
& \in\left\{\begin{array}{ll}
b, & g \\
a & o
\end{array}\right\}
\end{aligned}
$$

Thus $\{b, g, w\} \subset\left\{\begin{array}{ll}b & g \\ a & o\end{array}, w\right\}$. Hence and by Remark 5.2,

$$
-\{b, g, w\} \subset-\left\{\begin{array}{ll}
b, & g \\
a & o
\end{array}, w\right\}=\left\{\begin{array}{ll}
a, & o \\
b & g
\end{array}, w\right\}
$$

as well. Next observe that if the 1-morphism $a$ is the zero 1-morphism then $a H=1_{O}$ and $G a=1_{O}$ for any 2-morphisms $H$ and $G$. Now by definition an element of $\left\{\begin{array}{ll}b & g \\ 0 & 0\end{array}, w\right\}$ is of the form $-b K+S w+o H$ where in this case $K: 0 \Rightarrow g w, S: 0 \Rightarrow b g$ and $H: 0 \Rightarrow 0: W \rightarrow A$. But by the observation just made we have $o H=10: 0 \Rightarrow 0: W \rightarrow X$. Hence

$$
-b K+S w+o H=-b K+S w+1 o=-b K+S w \in\{b, g, w\}
$$

as claimed.

\section{REFERENCES}

1. M.G. Barratt, Homotopy operations and homotopy groups, AMS Summer Institute on Algebraic Topology, Seattle, 1963 (unpublished).

2. H.-J. Baues and W. Dreckmann, The cohomology of homotopy categories and the general linear group, K-Theory 3 (1989), 307-338. 
3. P. Gabriel and M. Zisman, Calculus of fractions and homotopy theory, Springer-Verlag, New York, 1967.

4. K.A. Hardie, K.H. Kamps and H.J. Marcum, Computing homotopy groups of a homotopy pullback, Quaestiones Math. 14 (1991), 179-199.

5. K. A. Hardie and A. V. Jansen, Toda brackets and the category of homotopy pairs, Quaestiones Math. 6 (1983), 107-128.

6. P. Hilton, Homotopy theory and duality, Gordon and Breach, New York, 1965.

7. H. J. Marcum, Parameter constructions in homotopy theory, An. Acad. Brasil. Ciênc. 48 (1976), 387-402.

8. _ Homotopy equivalences in 2-categories, Lecture Notes in Math, vol. 1425, Springer, 1990, pp. 71-86.

9. _ـ Functorial properties of the Hopf invariant (preprint).

10. M. Mimura, On the generalized Hopf homomorphism and the higher composition, Part I, J. Math. Kyoto Univ. 4 (1964), 171-190.

11. T. Müller, Zur Theorie der Würfelsätze, Dissertation, Fernuniversität Hagen, 1982.

12. H. Toda, Composition methods in homotopy groups of spheres, Ann. of Math. Stud., no. 49, Princeton Univ. Press, 1962.

Department of Mathematics, University of Cape Town, 7700 Rondebosch, SOUth Africa

E-mail address: hardiekaQuctrax.uct.ac.za

Fachbereich Mathematik, Fernuniversität, Postfach 940, D-58084 Hagen, Germany

The Ohio State University at Newark, 1179 University Drive, Newark, Ohio 43055

E-mail address: marcum@math.ohio-state.edu 